\title{
Electrooptical properties of GaNAs/GaAs multiple quantum well structures
}

\author{
Jia-Ren Lee ${ }^{\mathrm{a}}$, Yo-Yu Chen ${ }^{\mathrm{a}}$, Chien-Rong Lu ${ }^{\mathrm{a}, *}$, Wei-I Lee ${ }^{\mathrm{b}}$, Shih-Chang Lee ${ }^{\mathrm{b}}$ \\ ${ }^{a}$ Department of Physics, National Taiwan Normal University, Taipei 116, Taiwan, ROC \\ ${ }^{\mathrm{b}}$ Department of Electrophysics, National Chiao Tung University, Hsinchu 300, Taiwan, ROC
}

Received 12 December 2002; accepted 24 March 2003

\begin{abstract}
The electrooptical properties of the GaNAs/GaAs multiple quantum well structures have been studied using the photoreflectance spectroscopy from $20 \mathrm{~K}$ to room temperature. Above the band gap energy of GaAs, Franz-Keldysh oscillations were observed. The period of the Franz-Keldysh oscillations decreased slightly with decreasing temperature, and indicated that the corresponding space charge distribution varied slowly with temperature. The modulated quantum well transition features were observed below the band gap energy of GaAs. A matrix transfer algorithm was used to calculate the quantum well subband energies numerically. The band gap energy and the electron effective mass of the GaNAs/GaAs system were adjusted to obtain the subband energies to best fit the observed quantum well transition energies.
\end{abstract}

(C) 2003 Elsevier Science B.V. All rights reserved.

Keywords: Photoreflectance; GaNAs/GaAs; Quantum well

\section{Introduction}

Nitrogen containing III-V alloys such as GaNAs and GaInNAs are being studied intensively recently because of their interesting fundamental properties [1-8] and a wide range of possible optoelectronic applications [911]. Large bowing effect in combination with the possibility of obtaining a special lattice constant offer promises for major improvements in the performance and manufacturing of optoelectronic and photonic devices based on III-V nitrides. However, in spite of intensive studies, many of the fundamental parameters such as the bowing parameter, effective masses, the band alignment and the electronic states in the heterostructure systems are not well understood. There are still discrepancies among different experimental results and different theoretical predictions [12-20]. Further experimental explores are needed. This work reports the photoreflectance (PR) spectroscopy studies of the

* Corresponding author. Fax: +886-2-293-26408.

E-mail address: lupond@phy03.phy.ntnu.edu.tw (C.-R. Lu).
MOCVD grown GaNAs/GaAs multiple-quantum-well (MQW) structures at various temperatures.

\section{Experimental}

The samples were grown by MOCVD on the $\mathrm{n}^{+}$ doped GaAs substrate. After the undoped GaAs buffer layer, 20 periods of the $\mathrm{GaN}_{x} \mathrm{As}_{1-x} / \mathrm{GaAs}$ MQW structures were grown. The GaAs layers were all 25 $\mathrm{nm}$ thick. The nitrogen compositions of the $\mathrm{GaN}_{x} \mathrm{As}_{1-x}$ layers in different samples were varied up to $x=0.04$. The thicknesses were 10 or $6 \mathrm{~nm}$. A tungsten-halogen lamp with a monochromator provides the probing photons for the PR spectroscopy. The $514 \mathrm{~nm}$ line of an $\mathrm{Ar}^{+}$ion laser provides the pumping photons to generate free electron hole pairs to neutralize the space charge in the system and thus modulate the internal field strength. The pumping light was chopped at a frequency of $200 \mathrm{~Hz}$. Using the phase lock-in technique, the electro-modulated optical responses of the excitonic transition between the quantum well confined hole states and electron states are enhanced. The band edge transition exhibits Franz-Keldysh 
oscillatory (FKO) features whose period indicates the strength of the internal field [21-23]. These spectral characters make the PR experiment one of the best spectroscopic probes for the electrooptical properties.

\section{Results and discussion}

The PR spectra from GaNAs/GaAs MQW structures with different nitrogen compositions at $75 \mathrm{~K}$ are compared in Fig. 1. Pumping photons induced modulation of the internal electric field will result two types of electro-modulated line shape. When the internal electric field is weak, the modulated optical responses will be the derivative like line shape [23-27]. When the internal electric field is strong, the band edge transition will exhibit FKO features above the band gap energy. The FKO of Fig. 1 in the spectral range $h v>E_{\mathrm{g}}(\mathrm{GaAs})$, is originated from GaAs in the system. There are two types of GaAs regions in the system. Those are the GaAs layers in the MQW structure and the GaAs buffer/ substrate region. The MQW structure is undoped. The space charge density in the GaAs layers will not be high enough to induce strong internal electric field and to generate FKO. The electro-modulated spectral contributions from the MQW GaAs layers are those sharp derivative like excitonic features in Fig. 1 near $h v=E_{\mathrm{g}}$ (GaAs).

The FKO oscillatory features in the PR spectrum of Fig. 1 are caused by the internal electro-modulation in the GaAs buffer $/ \mathrm{n}^{+}$substrate region. The oscillation period indicates the strength of the internal field. The extrema in the FKO features of an electro-modulated spectrum are given by Refs. [24,28]

$n \pi=\phi+\frac{3}{4}\left[\frac{\left(E_{n}-E_{\mathrm{g}}\right)}{\hbar \Theta}\right]^{3 / 2}$

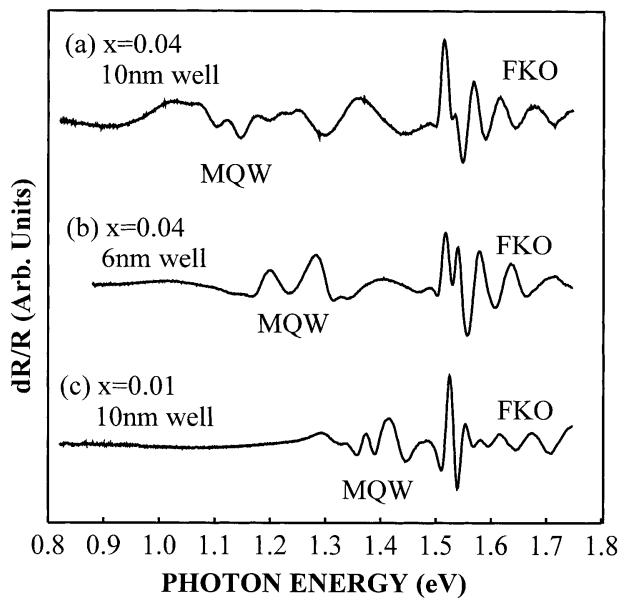

Fig. 1. Comparison of the $75 \mathrm{~K}$ PR spectra of the GaNAs/GaAs MQW structures with different well widths and nitrogen compositions. where $n$ is the index number of the extrema, $\phi$ is an arbitrary phase factor, $E_{n}$ is the photon energy of the $n$th oscillation extrema, and $E_{\mathrm{g}}$ is the band gap energy. The electrooptical energy $\hbar \Theta$ is defined by $(\hbar \Theta)^{3}=$ $e \hbar^{2} F^{2} /(2 \mu)$, where $F$ is the internal electric field, and $\mu$ is the reduced interband effective mass. A plot of $\left(E_{n}-\right.$ $\left.E_{\mathrm{g}}\right)^{3 / 2}$ versus $n$ yields a straight line with the slope proportional to the internal electric field. Fig. 2 shows the temperature dependence of the internal electric field in the GaAs region derived from the FKO features. The insert to the right in the figure is the FKO analysis, in which the data points fit better to a straight line in the higher index region because the asymptotic approximation is valid in the region $\left(E_{n}-E_{\mathrm{g}}\right) \gg \hbar \Theta$. The internal electric field originates from the spatial distribution of the space charge. In non-degenerate extrinsic semiconductors, lowering the temperature will tend to freeze out carriers, neutralize the space charge, and thus reduce the internal electric field. However, the observed internal electric field shown in Fig. 2 decreased only slightly as the temperature decreased. The Fermi level of the $n^{+}$ doped GaAs substrate is degenerate with the conduction band edge, and the Fermi level of the undoped GaAs buffer is close to the mid gap. They both varied slowly with temperature. The built in potential across the space charge distribution near the buffer/substrate $\left(\mathrm{i} / \mathrm{n}^{+}\right)$ interface will induce an internal electric field varying slowly with temperature as observed in Fig. 2.

Although the band gap energy of $\mathrm{GaN}$ is larger than that of GaAs, the $\mathrm{GaN}_{x} \mathrm{As}_{1-x}$ alloy shows a considerable red shift of the band edge with increasing nitrogen concentration for low $x$-values. Instead of being barriers, the $\mathrm{GaN}_{x} \mathrm{As}_{1-x}$ alloys become wells in the GaNAs/GaAs MQW structures under investigation. The contributions from the MQW GaNAs wells are those derivative like features labeled MQW in Fig. 1 in between $h v=E_{\mathrm{g}}$ (GaNAs) and $h v=E_{\mathrm{g}}$ (GaAs). Comparing spectra (a) and (b) from MQW structures with

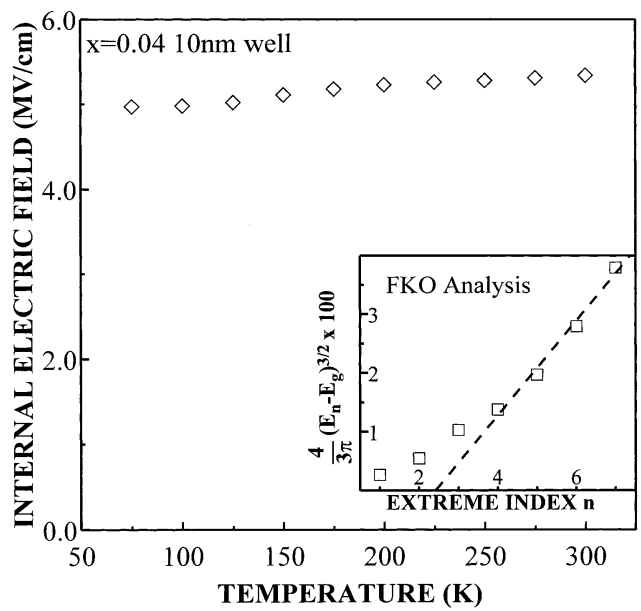

Fig. 2. The temperature dependence of the internal electric field in the GaAs region, and the insert to the right shows the FKO analysis. 
the same nitrogen composition $(x=0.04)$ but of different well width, the MQW transition features are blue shifted while the well width reduced from 10 to $6 \mathrm{~nm}$ as shown in Fig. 1. When nitrogen composition is reduced from $x=0.04$ to 0.01 as shown by spectra (a) and (c) in Fig. 1, the GaNAs band gap energy is increased, and the MQW transition features are also blue shifted. A matrix transfer algorithm [29] was used to calculate the MQW subband energies numerically. The wave functions of the $j$ th layer may be described by

$\varphi_{j}(z)=A_{j} e^{p_{j}(z)}+B_{j} e^{-p_{j}(z)}$,

where $A_{j}$ and $B_{j}$ are probability amplitudes and $p_{j}$ are phase factors. The cap and the buffer will be described by $j=0$ and 40 , respectively. Express the boundary conditions

$\varphi_{j-1}\left(z_{j-1}\right)=\varphi_{j}\left(z_{j}\right)$,

$\frac{1}{m_{j-1}^{*}} \frac{\mathrm{d}}{\mathrm{d} z}\left[\varphi_{j-1}\left(z_{j-1}\right)\right]=\frac{1}{m_{j}^{*}} \frac{\mathrm{d}}{\mathrm{d} z}\left[\varphi_{j}\left(z_{j}\right)\right]$,

in the matrix form

$$
\left[\begin{array}{l}
A_{j+1} \\
B_{j+1}
\end{array}\right]=M_{j}\left[\begin{array}{l}
A_{j} \\
B_{j}
\end{array}\right]
$$

where $M_{j}$ are the transfer matrices. The amplitudes of the top and the bottom barriers are related by

$$
\begin{aligned}
{\left[\begin{array}{l}
A_{40} \\
B_{40}
\end{array}\right] } & =M_{40} M_{39} \ldots M_{1} M_{0}\left[\begin{array}{l}
A_{0} \\
B_{0}
\end{array}\right] \\
& =\left[\begin{array}{ll}
\alpha_{11} & \alpha_{12} \\
\alpha_{21} & \alpha_{22}
\end{array}\right]\left[\begin{array}{l}
A_{0} \\
B_{0}
\end{array}\right] .
\end{aligned}
$$

For bound states, their amplitudes should converge outside the MQW region, and $A_{0}=0$ and $B_{40}=0$. Varying the energy in the MQW numerically, energies that make the matrix element $\alpha_{22}=0$ will be eigenenergies. The band gap energy, carrier effective mass, and the band-offset values were adjusted to obtain the GaNAs/GaAs MQW subband energies to fit the observed optical transition features. Most theoretical and experimental studies suggested that one of the nitrogen atomic levels mainly perturbs the conduction band, and its effect on the valence band is merely marginal [15-20]. The smallness of the valence band discontinuity in GaNAs/GaAs heterostructure leads to different band alignment results or even omission of the discontinuity [15-20]. X-ray photoelectron spectroscopy [18] and photoluminescence (PL) [19] studies suggested a slightly type-II band alignment with a small negative valence band-offset. Fig. 3 shows the $75 \mathrm{~K}$ PR spectrum of the GaNAs/GaAs MQW structures with $6 \mathrm{~nm}$ Ga$\mathrm{N}_{0.04} \mathrm{As}_{0.96}$ wells, and vertical bars in the figure indicate the calculated interband transitions. The symbols $11 \mathrm{H}$ (L) indicate the optical transitions involving the ground states of electron and heavy (light) hole, and $22 \mathrm{H}(\mathrm{L})$

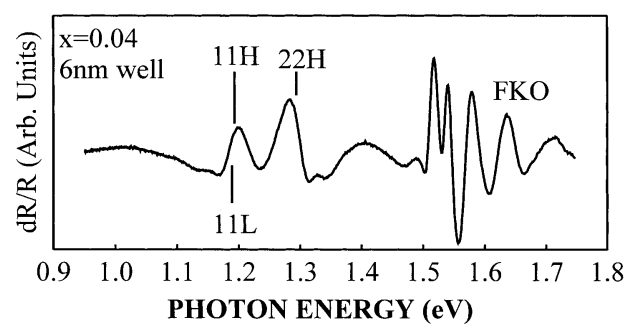

Fig. 3. $75 \mathrm{~K}$ PR spectrum of the GaNAs/GaAs MQW structure with 6 $\mathrm{nm} \mathrm{GaN} \mathrm{N}_{0.04} \mathrm{As}_{0.96}$ wells. The calculated MQW transitions involving the heavy hole are labeled on the top, and those involving light hole are labeled on the bottom.

indicate those involving the first excited states. The GaNAs layers in the GaNAs/GaAs MQW structures are under biaxial tensile strain, the band gap energies are smaller than the unstrained band gap of the bulk, and the heavy hole and light hole bands are split [30-32]. The electron-heavy hole ( $\mathrm{e}-\mathrm{hh})$ band gap energies were estimated from the $11 \mathrm{H}$ and $22 \mathrm{H}$ transition features in the PR spectra and the calculated MQW subband energies. The electron-light hole $(\mathrm{e}-\mathrm{lh})$ band gap energies were determined in reference to the splitting between the following empirical results [31].

$E_{\mathrm{e}-\mathrm{hh}}=0.99+0.43 \exp (-100 x / 1.87)$
$E_{\mathrm{e}-\mathrm{hh}}=0.86+0.56 \exp (-100 x / 2.51)$.

The band gap energy, band-offset value, and electron effective mass were adjusted to obtain the GaNAs/GaAs MQW subband energies to best fit the observed optical transition features. The strained band gap energies of $\mathrm{GaN}_{1-x} \mathrm{As}_{x}$ in the GaNAs/GaAs MQW structure deduced from PR spectra are summarized in Fig. 4. Comparing with those of strained thin films observed by PL [30,31], band gap energies deduced from PR spectra are slightly larger when the nitrogen content is higher.

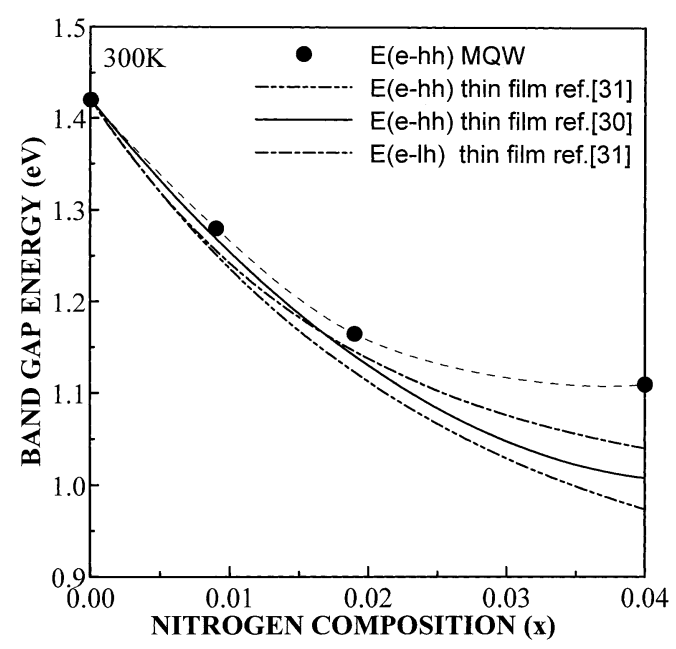

Fig. 4. The strained band gap energies of $\mathrm{GaN}_{1-x} \mathrm{As}_{x}$ at $300 \mathrm{~K}$ in the GaNAs/GaAs MQW structure are compared with those of strained films from Refs. [30,31]. 


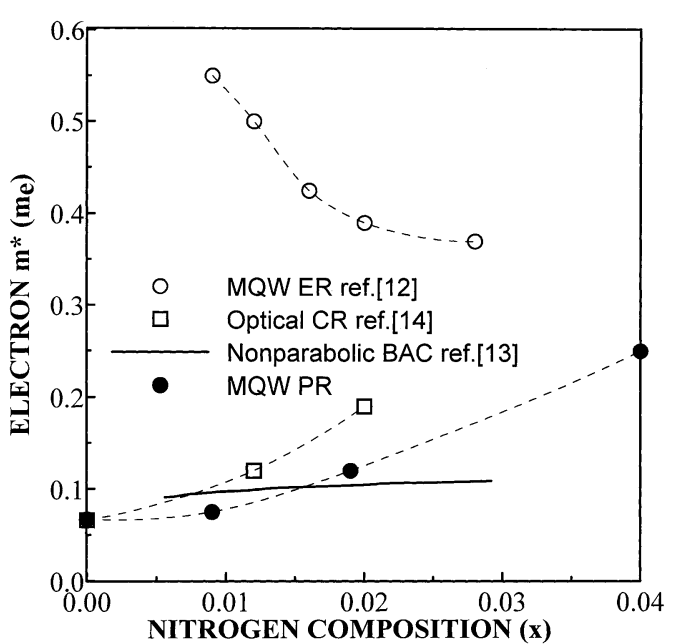

Fig. 5. The electron effective masses of the $\mathrm{GaN}_{1-x} \mathrm{As}_{x}$ in the GaNAs/GaAs MQW structure are compared with the those of elcetroreflectance (ER) studies [12], nonparabolic band anti-crossing (BAC) model [13] and optical cyclotron resonance (CR) [14].

The difference may be due to the nitrogen composition inhomogeneity induced band gap fluctuations in the GaNAs layers. PR is an excitation spectroscopy that can observe the mean band gap while PL emission is a recombination process that observes the band edge minimal. Fig. 5 shows the nitrogen composition dependence of the GaNAs electron effective mass. Previous electroreflectance study treated the GaNAs wells as heavily doped alloy with impurity band, assumed type-I valence band lineup, and deduced electron effective masses much larger than that of GaAs at first and reduced with further increase in nitrogen doping [12]. As shown in Fig. 5, the electron effective mass that is used in our GaNAs/GaAs MQW subband calculations are comparable to the results of nonparabolic band anticrossing model [13] and those of optical cyclotron resonance [14]. These fundamental parameters will be useful for further studies of the optoelectronic properties of the GaNAs heterostructure quantum systems.

\section{Summary}

Modulated optical responses of $\mathrm{GaN}_{x} \mathrm{As}_{1-x} / \mathrm{GaAs}$ MQW with different nitrogen compositions and well widths have been investigated by the PR spectroscopy at various temperatures. The origins of the electro-modulated features such as FKO and derivative like line shape are identified. The periods of the Franz-Keldysh oscillations were analyzed to extract the temperature dependence of the strength of the internal field. To study the quantum well confined electronic states, a matrix transfer algorithm was used to match the $\mathrm{GaN}_{x} \mathrm{As}_{1-x}$ I
GaAs boundary conditions and calculate the MQW subband energies numerically. The GaNAs band gap energies and electron effective masses were adjusted to obtain the GaNAs/GaAs MQW subband energies to best fit the observed transition features.

\section{References}

[1] M. Meyers, M. Sato, H. Ando, Jpn. J. Appl. Phys. Part 231 (1992) L853.

[2] T. Makimoto, H. Saito, T. Nishida, N. Kobayashi, Appl. Phys. Lett. 70 (1997) 2984.

[3] A. Zunger, Phys. Status Solidi B 216 (1999) 117.

[4] L. Bellaiche, S.H. Wei, A. Zunger, Phys. Rev. B 54 (1996) 17568.

[5] P.R.C. Kent, A. Zunger, Phys. Rev. Lett. 86 (2001) 2613.

[6] W. Shan, W. Walukiewicz, J.W. Ager III, E.E. Haller, J.F. Geisz, D.J. Friedman, J.M. Olson, S.R. Kurtz, Phys. Rev. Lett. 82 (1999) 1221.

[7] M. Hetterich, M.D. Dawson, A. Yu. Egorov, D. Bernklau, H. Riechert, Appl. Phys. Lett. 76 (2000) 1030.

[8] A. Lindsay, E.P. O’Reilly, Solid State Commun. 112 (1999) 443.

[9] C. Ellmers, F. Hohnsdorf, J. Hoch, C. Agert, S. Leu, D. Karaiskaj, M. Hoffman, W. Stolz, W.W. Ruhle, Appl. Phys. Lett. 74 (1999) 2271.

[10] M. Kondow, K. Uomi, A. Niwa, T. Kitatani, S. Watahiki, Y. Yazawa, Jpn. J. Appl. Phys. Part 135 (1996) 1273.

[11] G. Steinle, H. Riechert, A. Egorov, Electron. Lett. 37 (2001) 93.

[12] Y. Zhang, A. Mascarenhas, H.P. Xin, C.W. Tu, Phys. Rev. B 61 (2000) 7479.

[13] C. Skierbisxewski, S.P. Lepkowski, P. Perlin, T. Suski, W. Jantsch, J. Geisz, Physica E 13 (2002) 1078.

[14] P.N. Hai, W.M. Chen, I.A. Buyonva, H.P. Xin, C.W. Tu, Appl. Phy. Lett. 77 (2000) 1843.

[15] L. Bellaiche, S.H. Wei, A. Zunger, Phys. Rev. B 56 (1997) 10233.

[16] A. Lindsay, E.P. O'Reilly, Solid State Commun. 118 (2001) 313.

[17] S. Sakai, Y. Ueta, Y. Terauchi, Jpn. J. Appl. Phys. 32 (1993) 4413.

[18] T. Kitatani, M. Kondow, T. Kikawa, Y. Yazawa, M. Okai, K. Uomi, Jpn. J. Appl. Phys. Part 138 (1999) 5003.

[19] B.Q. Sun, D.S. Jiang, X.D. Luo, Z.Y. Xu, Z. Pan, L.H. Li, R.H. Wu, Appl. Phys. Lett. 76 (2000) 2862.

[20] J. Wu, W. Shan, W. Walukiewicz, K.M. Yu, J.W. Ager III, E.E. Haller, H.P. Xin, C.W. Tu, Phys. Rev. B 64 (2001) 85320

[21] W. Franz, Z. Naturforsh. 13 (1958) 484.

[22] L.V. Keldysh, Solv. Phys.-JEPT 7 (1958) 788.

[23] D.E. Aspnes, Surf. Sci. 37 (1973) 418.

[24] D.E. Aspnes, A.A. Studna, Phys. Rev. B 7 (1973) 4605.

[25] H. Shen, P. Parayanthal, F.H. Pollak, M. Tomkiewicz, T. Drommond, J.N. Schulman, Appl. Phys. Lett. 48 (1986) 653.

[26] B.V. Shanabrook, O.J. Glembocki, W.T. Beard, Phys. Rev. B 35 (1987) 2540.

[27] O.J. Glembocki, B.V. Shanabrook, Superlattices Microstruct. 5 (1989) 603.

[28] R.N. Bhattacharys, H. Shen, P. Parayanthal, F.H. Pollak, T. Coutts, H. Aharoni, Phys. Rev. B 37 (1988) 4044.

[29] M.O. Vassel, J. Lee, H.F. Lockwood, J. Appl. Phys. 54 (1983) 5206.

[30] U. Tisch, E. Finjman, J. Salzman, Appl. Phys. Lett. 81 (2002) 463.

[31] A.Y. Egorov, E.S. Semenova, V.M. Ustinov, Y.G. Hong, C. Tu, Semiconductors 36 (2002) 981.

[32] M.H. Ya, Y.F. Chen, Y.S. Huang, J. Appl. Phys. 92 (2002) 1446. 